\title{
Education and Training Needs, Methods, and Tools
}

\author{
P. Kotsampopoulos, T. V. Jensen, D. Babazadeh, T. I. Strasser $\mathbb{D}$, E. Rikos, \\ V. H. Nguyen, Q. T. Tran, R. Bhandia, E. Guillo-Sansano, K. Heussen, \\ A. Narayan, T. L. Nguyen, G. M. Burt, and N. Hatziargyriou
}

\section{Introduction}

A need for new skills and expertise to foster the energy transition has risen, considering the increased complexity of Cyber-Physical Energy Systems (CPES). Tackling the contemporary significant challenges requires a skilled workforce and researchers with systemic/holistic thinking and problem-solving skills. At the same time, technological advances can revolutionise education by allowing the use of new technical tools.

P. Kotsampopoulos $(\varangle) \cdot$ N. Hatziargyriou

National Technical University of Athens, Athens, Greece

e-mail: kotsa@power.ece.ntua.gr

T. V. Jensen $\cdot$ K. Heussen

Technical University of Denmark, Roskilde, Denmark

D. Babazadeh · A. Narayan

OFFIS - Institute for Information Technology, Oldenburg, Germany

T. I. Strasser

AIT Austrian Institute for Technology, Vienna, Austria

E. Rikos

Centre for Renewable Energy Sources and Saving, Athens, Greece

V. H. Nguyen

Université Grenoble Alpes, INES, Le Bourget du Lac, France

CEA, LITEN, Le Bourget du Lac, France

Q. T. Tran

Université Grenoble Alpes, INES, CEA, LITEN, Le Bourget du Lac, France

R. Bhandia

Delft University of Technology, Delft, The Netherlands

E. Guillo-Sansano · G. M. Burt

University of Strathclyde, Glasgow, Scotland, UK

T. L. Nguyen

Université Grenoble Alpes, Grenoble INP, Grenoble, France

(C) The Author(s) 2020

T. I. Strasser et al. (eds.), European Guide to Power System Testing,

https://doi.org/10.1007/978-3-030-42274-5_8 
In this framework, educational and training needs addressing the higher complexity of intelligent energy systems are identified in this chapter. State-of-the art laboratory-based and simulation-based tools are employed to address these needs. Real-time hardware in the loop simulation for hands-on laboratory education is applied and its benefits are explained. The learners gain access to remote labs, that allow the remote monitoring and control of laboratory facilities. Simulation-based tools that focus on co-simulation support the systemic understanding, while interactive notebooks promote problem-solving skills. Webinars and training schools allow the use of the proposed methods and tools by larger audiences and the collection of feedback. The developed material and tools are publicly available ${ }^{1}$ in order to promote the use and replicability of the approaches.

\section{Learning Needs for Modern Power and Energy Education}

Due to the increased complexity of intelligent power and energy systems, current and future engineers and researchers should have a broad understanding of topics of different domains, such as electric power, heat and definitely Information and Communication Technology (ICT) related topics. Appropriate education on modern topics is essential at university level, both for undergraduate and postgraduate studies, so that future engineers will be able to understand and tackle the challenges and propose/implement new methods. Recently, several universities have incorporated new courses in the undergraduate engineering curriculum or have enriched their existing courses with more modern material, while some universities have created dedicated master courses with relevant topics. The instruction is performed using traditional methods, such as class lectures, but also with programming, advanced simulations [25] and laboratory exercises [5, 10], that occasionally include the application of advanced learning methods such as problem-based learning and experiential learning $[6,14]$.

Moreover, the ongoing training of current professional engineers on modern topics is important. In some cases, professionals may tend to be hesitant of change and prefer to use proven technologies and methods. By taking part in proper training, professional engineers can better understand the benefits of modern solutions and ways to apply them in order to improve their daily work. Specifically, power system professionals frequently lack thorough understanding of ICT topics, while ICT professionals often find it hard to understand the operation of the power system. As these areas are closely connected due to the emergence of intelligent power and energy systems, it is important to create links between them. Obviously, a thorough understanding of all domains (electric power, heat, ICT, automation, etc.) is difficult to achieve, however an understanding of the fundamentals of each area, without sacrificing the expert focus in each particular field, will become increasingly important.

\footnotetext{
${ }^{1}$ https://erigrid.eu/education-training/.
} 
The same applies to researchers who are working to find solutions beyond the state of the art.

The future experts require the relevant insight and abilities to design and validate solutions for CPES. These abilities are facilitated by both generic engineering and cross-disciplinary technical competences. The generic competences include the conception, design, implementation and operation of systems (e.g. based on the Conceive Design Implement Operate (CDIO) skills catalogue [4]), which can be supported, for example, by project-oriented teaching methods. With increasing problem complexity, systems-oriented skills need to become strengthened, such as problem decomposition, abstraction and multi-disciplinary coordination of engineering challenges.

Cross-disciplinary learning is also required as the integration and interdependency of software and hardware systems is increased. Engineering students who aim to design and work with CPES solutions like complex control, data analytics, supervisory and decision support systems, require an increased level of programming and system design competences, as well as a pragmatic view on the applicability of methods. This means that some familiarity with domain specific system architectures and description methods is useful (e.g. reference architectures such as Smart Grid Architecture Model (SGAM) [8, 19]). Moreover, basic familiarity with distributed software system problems is important, as they are not addressed sufficiently within contemporary engineering education. In addition, simulation-based tools are useful for the emulation and understanding of physical behaviour and cyber-physical system couplings. More information on contemporary learning needs can be found in [12].

Summarizing, the following learning needs in the domain of intelligent power and energy systems are identified:

- Understanding the physical layer of CPES (especially topics related to distributed energy resources), including the interconnected sub-systems and components.

- Understanding automation and control systems.

- Understanding communication networks.

- Understanding optimization, data analytics and artificial intelligence.

- Understanding the mutual interactions/influences amongst components and domains.

Therefore, a holistic understanding of the physical and the cyber part of intelligent power and energy systems is necessary in order to design and develop a future reliable and sustainable energy system. This should be reflected in current and future education and training. In this direction, laboratory and simulation-based tools and methods are presented in the following sections to advance education and training in the smart grid era. 


\section{Laboratory Education}

Laboratory education provides a link between theory and real world offering valuable practical experience to students. In this section, new trends in laboratory education are presented, such as the use of real-time Hardware in the Loop (HIL) simulation and remote laboratories, including representative examples.

\subsection{Real-Time Simulation for Laboratory Education}

Laboratory education on power systems is usually performed with simulation software [7] or less frequently with dedicated hardware setups [15, 16, 20, 22]. On the other hand, laboratory education on power electronics and electric machines is typically performed with hands-on exercises using physical models or real hardware, as the focus is on the component level. The limited use of real hardware on power system education is obviously due to the difficulty and cost of having a realistic power system setup in the lab (including generators, transformers etc). As a result, small educational hardware setups usually perform specific functions and cannot be easily used for a wide range of experiments.

Real-time HIL simulation merges simulation and hardware testing providing hardware experience to the students, while exploiting the advantages of digital simulation. The following features of HIL simulation are beneficial for educational purposes:

- The students face a real-time system (like a SCADA), where they can perform actions and monitor the operation in realistic conditions. The flexibility, ease of modelling and designing test scenarios of digital simulation are maintained.

- The connection of real hardware devices such as inverters of Distributed Generation (DG), microgrids, relays can be realised, so that students can observe the operation of real apparatus. Measurement of actual magnitudes and control of real devices is a valuable experience.

- Components that are not available in the lab (e.g. transformer, diesel generator) can be simulated in real-time and their interaction with hardware devices can be studied.

- Challenging tests, such as faults, can be performed safely and conveniently in a real-time simulation environment without hazardous effects or equipment stress. The type, duration and location of faults can be easily modified by the students, which would be difficult in a real hardware setup.

Controller Hardware-in-the-Loop (CHIL) simulation has been used several times for laboratory education [3, 24], however the potential of Power Harware-in-theLoop (PHIL) experiments could be perfectly explored during the ERIGrid project since it required a new and more complex technical approach. 


\subsubsection{Laboratory Exercise Examples}

The laboratory exercises aim to introduce university students to the world of real-time simulation and its various applications in the power system domain. Several modules have been created which start from an introduction to real-time simulation, leading to more advanced modelling and interfacing techniques, using a hands-on approach. At first, the students become familiar with real-time simulation software (RSCAD of RTDS) by executing several examples. Interfacing with other software tools is introduced (e.g. Matlab and Python), highlighting the co-simulation possibilities. The possibility of developing custom component models and the small time-step modelling of power electronic devices are explained. Next, the use of communication protocols (e.g. IEC 61850) is highlighted, along with interfacing techniques with hardware equipment. Finally, practical examples of modelling of Photovoltaic (PV) systems and High Voltage DC (HVDC) links are provided.

A laboratory exercise for explaining voltage control of distribution networks with distributed generation is discussed in detail, where the students have the opportunity to monitor and control actual equipment. An overview of the PHIL/CHIL setup of the experiment is shown in Fig. 1. A hardware PV inverter and a load bank are connected to a simulated weak distribution network fed by a transformer equipped with an OnLoad Tap Changer (OLTC). The students control the active power of the PV simulator by changing the irradiation for a given I-V curve via its software environment. While keeping the load low, they steadily increase the active power of the PVs from zero to nominal and observe the voltage rise occurring at the inverter's terminal. The students try to solve this overvoltage problem and suggest as a solution the reactive power absorption by the PV inverter. They send reactive power absorption set-points to the PV inverter via its software interface, monitor the voltage and validate its effect. In this way, the need for DG to support the grid by providing ancillary services is highlighted. More complex solutions are gradually demonstrated and explained, such as the application of coordinated voltage control that requires the existence of a telecommunication network, the solving of an optimization problem etc.

The laboratory exercises are designed according to the principles of experiential learning, based on Kolb's four-stage learning cycle [11]. According to that cycle, the initial concrete experience is being elaborated and reflected upon (reflective observation) to enable the learner to reach an abstract conceptualization, which is the third stage of the learning cycle. That abstract concept is being applied in real life situations (active experimentation) so that a new concrete experience emerges which is elaborated and reflected upon and so on. Accordingly, during the classroom lectures the students are taught fundamentals of power system operation. During the experiments, the DG integration topics are offered to the students directly in the lab (concrete experience) without in-depth theoretical knowledge. After each experiment, suitable questions on real problems are posed to facilitate understanding, taking into account the student's existing knowledge (reflective observation). Guided conversations with the students or direct instruction, when considered necessary, lead to new concepts (abstract conceptualization). On this ground, new experiments are performed (active experimentation and new concrete experience). The reports at the 


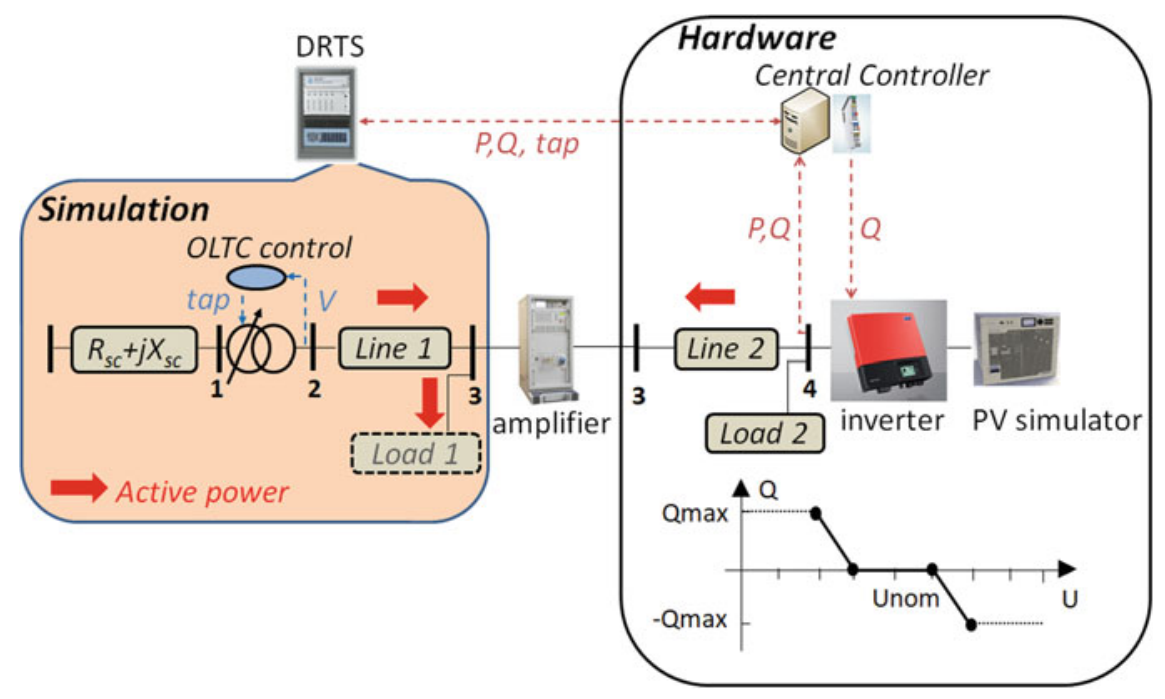

Fig. 1 Voltage control by DG, OLTC and centralised coordinated control (PHIL and CHIL simulation) [14]

end of the session aim to reflect the laboratory experience (reflective observation). More information on the laboratory exercises can be found in [14].

\subsection{Remote Laboratories}

Remote labs are gaining significant attention for educational purposes, as they allow the user to connect remotely to actual laboratory infrastructure, obtain measurements and control devices $[1,9]$. Two remote laboratory applications are presented next.

\subsubsection{Voltage Control}

The remote lab for voltage control provides online access to actual laboratory equipment, allowing measurement and control via the laboratory SCADA. The laboratory setup includes a PV inverter that is connected to the utility grid via a long low voltage line. The user can control the active and reactive power of the inverter and monitor the resulting voltage at the actual hardware setup, from the web-based Graphical User Interface (GUI), shown in Fig. 2.

Moreover, a virtual lab has been developed that uses a mathematical representation of the system and is also available as a web-based tool. The remote lab is more realistic than the virtual lab, as the operation of the real system is observed, providing a more meaningful experience to the user. In addition, phenomena such as noise, equipment 


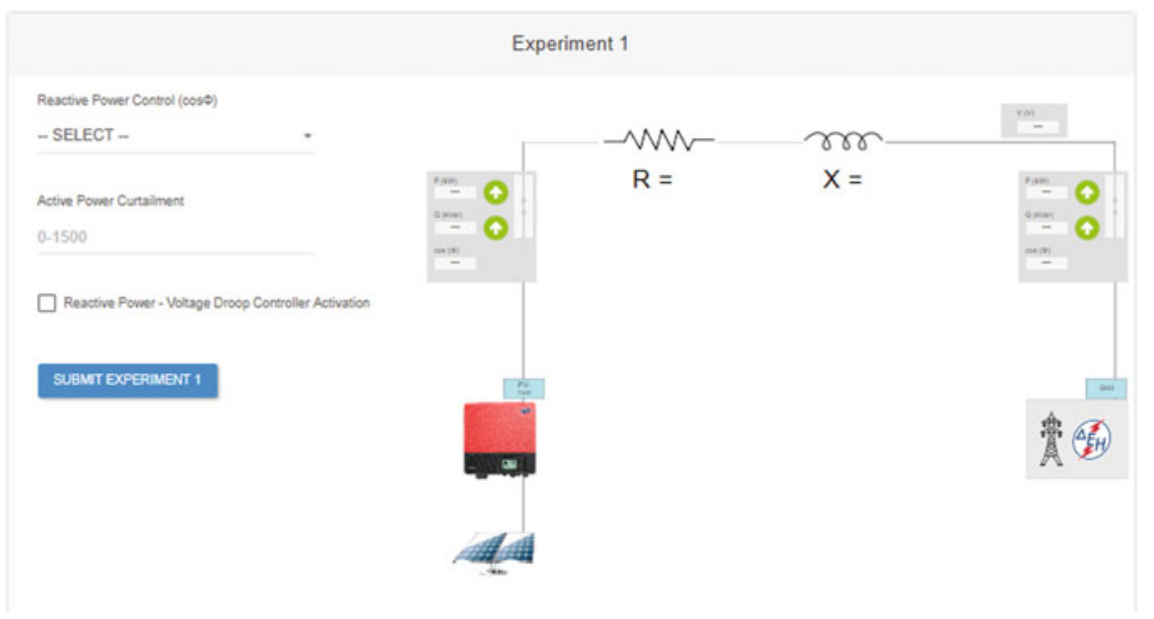

Fig. 2 Graphical user interface of the remote lab for the voltage control experiment

inaccuracies, communication delays, etc which are inherent in a real-life system are taken into account at the remote lab. On the other hand, the use of the remote lab presents some challenges. Most importantly, only one user can typically have access to the remote lab application at a time (because there is only one laboratory setup available to control), whereas the virtual lab can be used by simultaneously by a large number of users at the same time through the online platform. Therefore, it is more difficult to offer the remote lab to a wide audience. Moreover, for safety reasons it is recommended that laboratory staff monitors the process of the experiment and communicates with the user if necessary.

\subsubsection{Microgrid Balancing}

In order to facilitate the understanding of concepts such as multi-domain experiments, interoperability of control devices and real-time simulation of physical components, the Microgrid Balancing remote lab application was designed and implemented. The basic idea of this experiment is that a user can remotely connect to the experimental microgrid and interact with the SCADA system in order to achieve a power balancing operation based on specific market policy. In the setup the battery, load, and grid connection are all physical components, whereas the PV unit is simulated (in MATLAB/Simulink) in order to introduce the analytical mathematical models of the PV system (e.g. calculation of I-V characteristic, Maximum Power Point, injected $\mathrm{AC}$ power). The input signals to the PV model are global horizontal irradiance and ambient temperature from real-time measurements. Figure 3 provides an overview of the Simulink model blocks. 


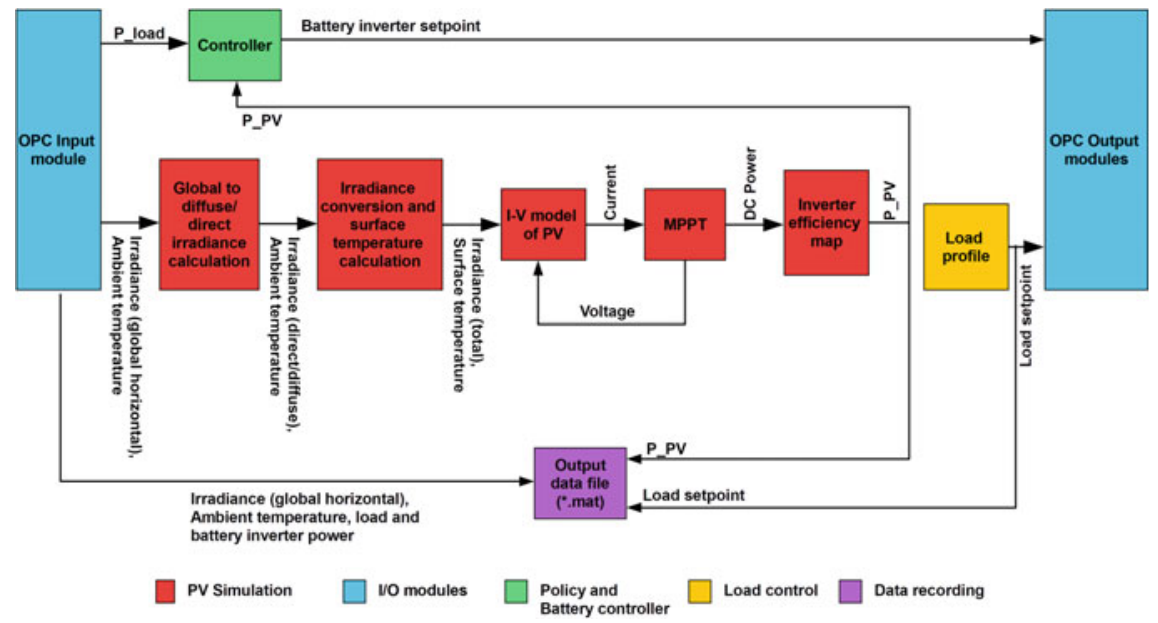

Fig. 3 Analytical illustration of the control blocks of the remote lab for microgrid balancing

The controller makes use of a simple strategy which chooses a power set-point for the batteries according to the power values of the PV and loads. The control is divided into two scenarios, named as "sell" and "buy priority". In "sell priority" the injection of the PV power surplus to the grid is prioritised, while in "buy priority" the battery covers the power imbalance by absorbing any PV power surplus.

\section{Simulation-Based Tools}

Given the cross-disciplinary nature of intelligent power and energy systems as outlined above, students should be exposed to a wide set of tools and concepts related to different domains. Thus, new educational methods and tools must be developed, capable of bringing the knowledge of different domains together and allowing the students to understand the coupling and interaction of elements within intelligent solutions.

It is clear that simulations will play an important role in the design, analysis and testing process of new solutions. It is therefore natural that students should learn to use domain-specific simulation tools, both in standalone and in co-simulation setups. The students should also understand the limitations of such tools. Moreover, methods that support students to bridge the gap of theory and application are required. 


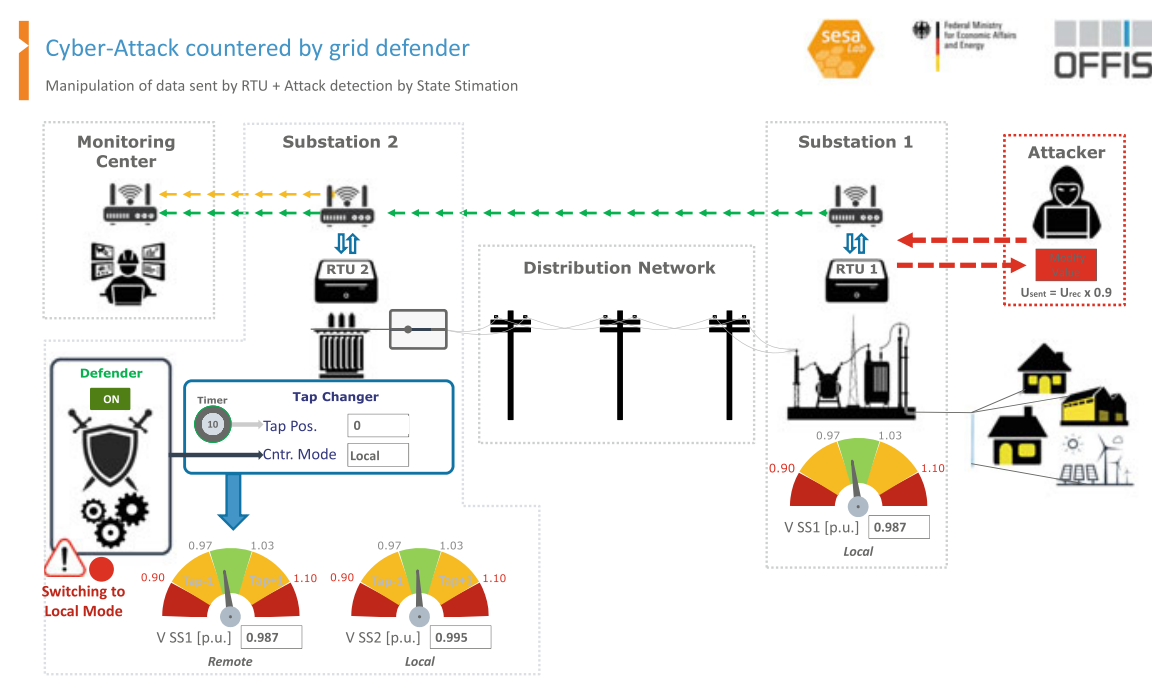

Fig. 4 Graphical user interface of the cyber-resilience tool

\subsection{Co-simulation Tools}

\subsubsection{Mosaik-Based Co-simulation}

The goal of mosaik co-simulation framework is to provide researchers with an easyto-use yet powerful tool for simulation-based testing [21, 23]. It provides a flat learning curve for learners/researchers wishing to test their simulations in common scenarios with others, allowing intuitive co-modelling of various power system scenarios. Next to its research applications, mosaik is used for training and education in the field of CPES. In a practical course, students learn to plan, execute, and analyse co-simulation-based experiments. The target audience includes researchers and students from domains such as computer science, environmental modelling and renewable energy. They learn how to develop models of electrical components and integrate them into smart grid scenarios. They also learn to develop distributed agent-based control algorithms for smart grids. These developments can be done in individual modelling environments and are co-simulated using mosaik. The goal is to analyze the requirements for real-time performance, accuracy, resource utilization and the reliability of the simulation results.

\subsubsection{Cyber-Resilience Tool}

The increased penetration of active components and digitalization intensifies the system complexity, resulting in higher risk of ICT incidents [18], thereby expand- 
ing the scope of cyber threats. The Cyber-Resilience Tool is an educational tool to demonstrate how cyber vulnerabilities could affect an electrical distribution grid. It also shows a possible defensive action against cyber-attacks. The tool has a GUI (shown in Fig. 4) with which the users can perform certain attacks and investigate their impact on the system; both with and without defensive measures. Since smart grids consists of multiple domains, one of the main challenges is the integration of different tools i.e. co-simulation, so as to analyse different domains. The tool consists of a real-time simulation environment including the following components:

- Modelling of the power system feeder, e.g. loads, lines, busbars and transformer (ePHASORSIM from OPAL-RT).

- Modelling of the tap changer controller of the transformer and the defensive mechanism of the system (eMEGASIM from OPAL-RT).

- Modelling of the communication infrastructure between the substations using IEC 60870-5-104 protocol (EXata from Scalable Network Technologies).

- Communication protocol translation and modelling of payload alteration options, i.e. cyber-attacker (Virtual Remote Terminal Unit (vRTU) from OFFIS).

\subsubsection{FMU-as-a-Service Approach}

The Functional Mock-up Interface (FMI) is a standard in co-simulation that allows interoperability among models from different domains. The Functional Mock-up Unit (FMU - the basic brick of FMI standard) encloses the dynamic model and generates compiled $\mathrm{C}$ code, which can be integrated into other environments as a black box. The deployment of FMUs in a co-simulation framework is however problematic for novice users without extensive informatics background. This limits the development of FMU and hinders the learning curve as well as the utilization of FMI by new users.

In this context, a software tool has been developed allowing the delivery of FMU in a Software-as-a-service manner, named as FMU-as-a-service. The server is developed on Django with PyFMI as the solver. Results are available in JSON-CSV-HTML or can be represented at the graphical web interface. With the proposed tool, no installation is required from the user side, and an FMU could be executed without further requirements (e.g. toolbox, solver request, etc.). Moreover, the platform allows multiple users working on the same model at the same time. The user can also deposit and encrypt (RSA) their FMU model to the server to keep the code confidential.

One of the main objectives of this software is to help students and interested users to understand the functionality and structure of a functional mock-up unit and to become familiar with planning, executing and evaluating simulation-based experiments. Initially developed to serve only in the smart building validation domain, it can be used for various courses involving (co)simulation, such as mechatronics or robotics or eventually complex cyber-physical systems. Moreover, the software can be used to provide a comprehensive course on different type of simulations, methods of computation and on their interaction. 


\subsection{Interactive (Jupyter) Notebooks}

A main issue with smart grid validation, especially for large-scale systems, is the complexity of the resulting simulation. When instructing in these complex simulations, a large portion of time is spent trying to couple the overall learning of the activity into the code that students must write or engage with. As the complexity of the code base inevitably increases, students who are not used to dealing with this aspect may feel lost in the aspects of the code itself. In the worst case, this may cause the student to miss the high-level learning involved. One way to address this issue is to apply interactive notebooks in instruction.

Jupyter notebooks are a merge between a standard text book and what real programming in python looks like [2]. The notebooks can be built from explanatory text and figures, while a full Python kernel allows the student to execute python code. The use of these notebooks provides a way to narrow the gap between theoretical concepts and application by setting up code examples, where the student is able to directly see examples of the theoretical concepts explained in the text. Notebooks of this kind can be developed to cover a wide spectrum of intelligent energy systems concepts.

By constructing a framework where students can focus on a problem to solve, instead of dealing with issues related to programming, they are able to better absorb and understand the core course concepts. The learning objectives of the developed notebooks are as follows:

- Design a testing procedure to validate a "black box" algorithm.

- Recognize the importance of statistical Design of Experiments to qualifying tests.

- Apply Design of Experiments to evaluate the performance of a "black box" algorithm.

As an example of the application of this tool, a pair of notebooks were given to the students for hands-on experience with a co-simulation environment and Design of Experiments [17]. The example used consisted of a typical Home Energy Management System in which a scenario is built up step by step, to include a house with solar panels, battery and a controller that can connect the house to the grid or discharge the battery. All the components and interconnections were modelled in behind-the-scenes python scripts and the simulation ran using the python module mosaik. An example of the final notebook can be seen in Fig. 5.

\section{Outreach Activities}

Outreach educational/training activities, such as the delivery of webinars, training schools and workshops promote the use of the proposed methods and tools by larger audiences. Insights from the delivery of several webinars, training schools and workshops are provided below. 


\subsection{Webinars}

The use of webinars (i.e. web seminars) as an e-learning environment is receiving more and more attention [26, 27]. The possibility of addressing large audiences, conducting live exercises/experiments to engage with the audience and also interacting with the presenters through questions and answers, render webinars a valuable educational tool. Moreover, the activity presented at the webinar is typically recorded and distributed to the participants and also made publicly available through video sharing platforms for later viewings at the learner's own pace. In this way a larger audience than the initial participants are able to benefit from the webinar.

In the contemporary cyber-physical environment, when the learning topics are related to the introduction of complex methods and tools (e.g. advanced testing and simulation), the webinar is an efficient option to achieve the learning objectives. For example, cases in which a webinar was found to be an effective learning environment are:

1. Introduction to a new software tool, where the participants have no previous experience.

2. Performance of live demonstrations of simulations and also laboratory tests.

3. Understanding co-simulation possibilities for cyber-physical systems.

It should be noted that feedback from the webinar participants can be easily obtained and analysed in order to improve the learning process.

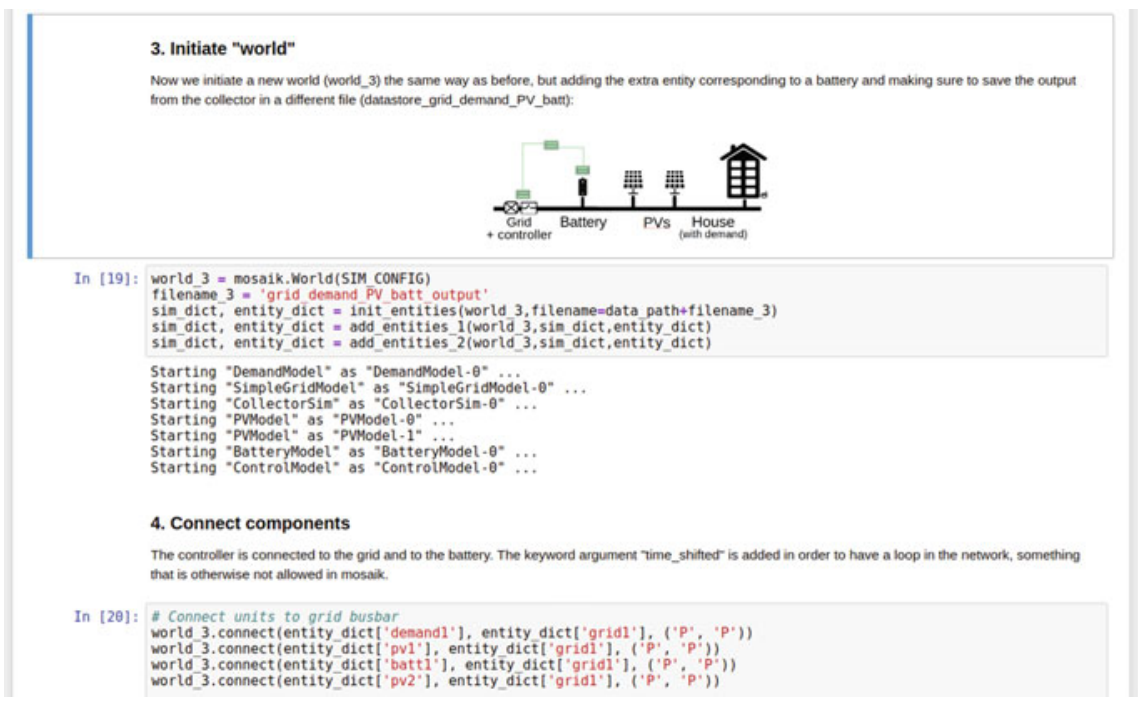

Fig. 5 Example of a Jupyter notebook, where markup language and Python code can be used to showcase complex examples 


\subsection{Training Schools and Workshops}

Training schools, such as summer schools or winter schools, typically target $\mathrm{PhD}$ candidates, early stage researchers, and young professionals and have a duration that allows the in-depth analysis of the given topic. On the other hand, a workshop has a shorter duration and is typically focused on giving necessary background for application. While a training school or workshop is necessarily limited in the scope of the audience that can benefit from it, a main advantage is that the participants can have close interaction with the instructors. Thus, training schools and workshops are particularly relevant when the learning goal is to:

- Familiarise with the use of a local laboratory infrastructure.

- Apply advanced theoretical methods, especially when few standard learning resources exist for the smart grid context.

- Familiarize with complex co-simulation methods.

- Design and evaluate systems for which the failure modes are too numerous to anticipate.

As laboratory-based methods play an important role in the analysis of CPES, training schools and workshops proved to be efficient educational/training settings. Hands-on approaches were applied which included individual work (e.g. familiarization with a specific software) combined with team work (e.g. joint modelling, fine tuning and running simple experiments). Live demonstrations are beneficial when an experiment is too complex for a hands-on activity and can better demonstrate the capabilities and inspire the participants. A well-balanced set of lectures, hands-on laboratory work and visits to industrial installations was found to be an ideal combination for a successful event, as long as the activities integrate in a structured way into the event's overall purpose.

\section{Conclusions}

The emergence of intelligent solutions in the domain of power and energy systems opens new possibilities but poses new challenges, rendering appropriate education and training methods for students and engineers increasingly important. A broad understanding of several domains is necessary to deal with the increased complexity and diversity, including electric power systems, automation, ICT, thermal systems etc. This chapter identifies upcoming educational needs and requirements in this rising complex environment. It is explained that the validation of complex systems is a multi-stage process, while systems-oriented skills and cross-disciplinary learning needs to be cultivated. In order to cover the distance between theory and handson practice, coding and laboratory education is beneficial. As the required field of knowledge is too broad, educational methods such as experiential learning and 
problem-based learning can prove to complement effectively the traditional teaching methods.

In this framework, state of the art laboratory-based and simulation based-tools and methods were presented, accompanied by representative examples. The benefits of real-time simulation for educational purposes were clearly explained, highlighting the provision of hands-on experience to students. The remote labs give users the possibility to experience lab conditions by gaining online access to actual laboratory installations providing a realistic experience. Simulation-based tools are efficient ways to educate on CPES topics such as co-simulation. They can help bridging the gap between theory and application, can be used in different settings (e.g. classroom sessions, e-learning, demonstrations at workshops) and support blended learning. The delivery of webinars and the organization of training schools proved to be an effective way to educate students, researchers and professionals on emerging topics. It is shown that a holistic approach using advanced tools and methods can advance education and training in the field of CPES. More information about the developed tools and methods can be found in [13].

\section{References}

1. Aydogmus, Z., Aydogmus, O.: A web-based remote access laboratory using scada. IEEE Trans. Educ. 52(1), 126-132 (2008)

2. Cardoso, A., Leitão, J., Teixeira, C.: Using the Jupyter notebook as a tool to support the teaching and learning processes in engineering courses. In: International Conference on Interactive Collaborative Learning, pp. 227-236. Springer (2018)

3. Celeita, D., Hernandez, M., Ramos, G., Penafiel, N., Rangel, M., Bernal, J.D.: Implementation of an educational real-time platform for relaying automation on smart grids. Electr. Power Syst. Res. 130, 156-166 (2016)

4. Crawley, E.F., Malmqvist, J., Lucas, W.A., Brodeur, D.R.: The CDIO syllabus v2. 0. an updated statement of goals for engineering education. In: Proceedings of 7th International CDIO Conference, Copenhagen, Denmark (2011)

5. Deese, A.S.: Development of smart electric power system (seps) laboratory for advanced research and undergraduate education. IEEE Trans. Power Syst. 30(3), 1279-1287 (2014)

6. Felder, R.M., Brent, R.: The abc's of engineering education: Abet, bloom's taxonomy, cooperative learning, and so on. In: Proceedings of the 2004 American Society for Engineering Education Annual Conference and Exposition, vol. 1 (2004)

7. Georgilakis, P.S., Orfanos, G.A., Hatziargyriou, N.D.: Computer-assisted interactive learning for teaching transmission pricing methodologies. IEEE Trans. Power Syst. 29(4), 1972-1980 (2014)

8. Gottschalk, M., Uslar, M., Delfs, C.: The Use Case and Smart Grid Architecture Model Approach: The IEC 62559-2 Use Case Template and the SGAM Applied in Various Domains. Springer, Berlin (2017)

9. Gustavsson, I.: Remote laboratory experiments in electrical engineering education. In: Proceedings of the Fourth IEEE International Caracas Conference on Devices, Circuits and Systems (Cat. No. 02TH8611), pp. I025-I025 (2002)

10. Hu, Q., Li, F., Chen, C.F.: A smart home test bed for undergraduate education to bridge the curriculum gap from traditional power systems to modernized smart grids. IEEE Trans. Educ. 58(1), 32-38 (2014) 
11. Kolb, D.A.: Experiential Learning: Experience as the Source of Learning and Development. FT Press (2014)

12. Kotsampopoulos, P., Hatziargyriou, N., Strasser, T.I., Moyo, C., et al.: Validating intelligent power and energy systems - a discussion of educational needs. In: Marik, V., Wahlster, W., Strasser, T., Kadera, P. (eds.) Industrial Applications of Holonic and Multi-Agent Systems. HoloMAS 2017. Lecture Notes in Computer Science, vol 10444. Springer, Cham (2017)

13. Kotsampopoulos, P., Maniatopoulos, M., Tekelis, G., Kouveliotis-Lysikatos, I., et al.: D-NA4.2a Training/education material and organization of webinars. Deliverable D4.3, ERIGrid Consortium (2018)

14. Kotsampopoulos, P.C., Kleftakis, V.A., Hatziargyriou, N.D.: Laboratory education of modern power systems using phil simulation. IEEE Trans. Power Syst. 32(5), 3992-4001 (2016)

15. Kuzle, I., Havelka, J., Pandžić, H., Capuder, T.: Hands-on laboratory course for future power system experts. IEEE Trans. Power Syst. 29(4), 1963-1971 (2014)

16. Maza-Ortega, J.M., Barragán-Villarejo, M., de Paula García-López, F., Jiménez, J., Mauricio, J.M., Alvarado-Barrios, L., Gómez-Expósito, A.: A multi-platform lab for teaching and research in active distribution networks. IEEE Trans. Power Syst. 32(6), 4861-4870 (2017)

17. van der Meer, A.A., Steinbrink, C., Heussen, K., Morales Bondy, D.E., et al.: Design of experiments aided holistic testing of cyber-physical energy systems. In: 2018 Workshop on Modeling and Simulation of Cyber-Physical Energy Systems (MSCPES), pp. 1-7. IEEE (2018)

18. Narayan, A., Klaes, M., Babazadeh, D., Lehnhoff, S., Rehtanz, C.: First approach for a multidimensional state classification for ICT-reliant energy systems. In: International ETG-Congress 2019; ETG Symposium, pp. 1-6. VDE (2019)

19. Neureiter, C., Engel, D., Trefke, J., Santodomingo, R., Rohjans, S., Uslar, M.: Towards consistent smart grid architecture tool support: from use cases to visualization. In: IEEE PES Innovative Smart Grid Technologies, Europe, pp. 1-6 (2014)

20. Rasheduzzaman, M., Chowdhury, B.H., Bhaskara, S.: Converting an old machines lab into a functioning power network with a microgrid for education. IEEE Trans. Power Syst. 29(4), 1952-1962 (2014)

21. Rohjans, S., Lehnhoff, S., Schütte, S., Scherfke, S., Hussain, S.: Mosaik-a modular platform for the evaluation of agent-based smart grid control. In: IEEE PES ISGT Europe 2013, pp. 1-5. IEEE (2013)

22. Santoso, S., Lwin, M., Ramos, J., Singh, M., Muljadi, E., Jonkman, J.: Designing and integrating wind power laboratory experiments in power and energy systems courses. IEEE Trans. Power Syst. 29(4), 1944-1951 (2014)

23. Schütte, S., Scherfke, S., Tröschel, M.: Mosaik: A framework for modular simulation of active components in smart grids. In: 2011 IEEE First International Workshop on Smart Grid Modeling and Simulation (SGMS), pp. 55-60. IEEE (2011)

24. Srivastava, A., Schulz, N.: Applications of real time digital simulator in power system education and research. In: American Society for Engineering Education. American Society for Engineering Education (2009)

25. Strasser, T., Stifter, M., Andrén, F., Palensky, P.: Co-simulation training platform for smart grids. IEEE Trans. Power Syst. 29(4), 1989-1997 (2014)

26. Verma, A., Singh, A.: Leveraging webinar for student learning. In: 2009 International Workshop on Technology for Education, pp. 86-90 (2009)

27. Wang, S.K., Hsu, H.Y.: Use of the webinar tool (elluminate) to support training: The effects of webinar-learning implementation from student-trainers' perspective. J. Interact. Online Learn. 7(3), 175-194 (2008) 
Open Access This chapter is licensed under the terms of the Creative Commons Attribution 4.0 International License (http://creativecommons.org/licenses/by/4.0/), which permits use, sharing, adaptation, distribution and reproduction in any medium or format, as long as you give appropriate credit to the original author(s) and the source, provide a link to the Creative Commons license and indicate if changes were made.

The images or other third party material in this chapter are included in the chapter's Creative Commons license, unless indicated otherwise in a credit line to the material. If material is not included in the chapter's Creative Commons license and your intended use is not permitted by statutory regulation or exceeds the permitted use, you will need to obtain permission directly from the copyright holder. 